\title{
Understanding prism adaptation: An individual differences approach
}

\author{
DAVID H. WARREN and BRUCE B. PLATT \\ University of Califormia, Riverside, Califormia 92502
}

\begin{abstract}
Adaptation of college students to wedge prisms was studied in terminal exposure and continuous exposure conditions. The adaptation experience was preceded by the administration of a battery of pretests designed to evaluate several visual and motor abilities thought to be involved in adaptation performance. Ability indices were formed for each subject on the basis of his performance on the pretests. These indices were then used in multiple regression analyses with several measures of adaptation as dependent variables. The ability indices accounted for substantial portions of the dependent measure variance. In the terminal exposure condition, amount of adaptation was positively correlated with indices of eye ability and negatively correlated with indices of hand ability. Intermanual transfer of adaptation was positively correlated with hand abilities and negatively correlated with eye abilities. In the continuous exposure condition, amount of adaptation was positively correlated with eye abilities and negatively correlated with hand abilities. It was suggested that theoretical and experimental approaches may be oversimplified if they do not take into account the possibility that at least some of the differences in the adaptation shown by different subjects is attributable to differences in abilities that are basic to the process of adaptation.
\end{abstract}

Studies of adaptation to the lateral visual displacement produced by wedge prisms have typically found substantial between-subject variability. Under identical experimental conditions, some subjects adapt very little, while others adapt almost completely. There has been very little serious attention paid to this variability - it has routinely been treated as error variance, either explicitly or implicitly. At the same time, there has been a great deal of attention paid to the theoretical controversy over whether adaptation consists of a shift in perceived visual position or in felt position of the hand or arm. Harris (1965) has served as a major spokesman for the hand shift hypothesis, while McLaughlin, Rifkin, and Webster (1966) provide an example of the visual shift hypothesis.

These two topics, response variability and visual vs. hand shift hypothesis, may be more related than is apparent at first glance. If adaptation consists of two or more components (e.g., both visuomotor and proprioceptive hand shifts), then it may be possible to account for some of the variability in adaptation results by taking between-subjects differences into

The research was supported by a University of California Intramutal Research Grant to the first author. Part of the matcrial was presented at the Conference on the Recombination Procediure as a Toul for the Study of Visual Perception. November 1973. at the University of Kansas. We thank Profiessur Roy D. Goldman for his patient statistical consultation and we thank Steve Morrell, Jill Osborn. David Rapaport. lirm Sclumitt, and David Huttner for their long hours of rescaircle alssinance. Send requests for reprints to David $H$. Warren. Department of Psychoiogy, University of California, Riverside. Cillitornia 92502. account. Specifically, it may be useful to consider abilities that might lead subjects to experience different anounts of visuomotor and proprioceptive hand shifts.

There is evidence that adaptation to the lateral displacement produced by prisms consists of at least two components. In a long-term study, Hay and Pick (i966) found that a proprioceptive shift occurred in the early part of the exposure period, and that an oculomotor shift gradually appeared, replacing the proprioceptive shift. McLaughlin and Bower (1965) provided evidence for the concurrent development of oculonotor and proprioceptive hand-shift components. The oculomotor component produced a shift in the responses of both exposed and nonexposed hands, while the proprioceptive hand shift occurred only for the exposed hand. McLaughlin et al. (1966) provided a similar demonstration that both proprioceptive hand-shift and oculomotor components of adaptation occur at the same time. Further, the oculomotor component was separable into two types, one dependent on perceived lateral shift and the other dependent on the apparent rotation of a plane perpendicular to the line of sight. Welch and Rhoades (1969) measured the magnitude of negative aftereffect (NA) and of proprioceptive shift (PS) under various information conditions in an attempt to determine what sources of information were used in producing each type of adaptation. Their results supported the notion that at least two types of adaptation occur simultaneously in the same subjects: "NA and PS represent qualitatively different kinds of adaptation. since they are neither highly correlated with each 
other nor affected in the same manner by the manipulation of informational variables" (p.425). Thus it is evident that adaptation to the lateral displacement produced by prisms consists of two or more components, and that the different components depend on different sources of information.

The experiments reported here were designed to investigate the possibility that some between-subjects variability in adaptation may be explained by taking into account certain perceptual abilities of the individual subjects. Specifically, the exact way that a subject uses the available visual and proprioceptive information should depend on his visual and hand abilities, and so the characteristics of his adaptation performance should also depend on his visual and manual abilities. Assessment of the relevant abilities should therefore allow a more complete explanation of adaptation results.

Two experiments were conducted with separate samples of college students. In the first experiment, terminal exposure was provided. The subject viewed a visual target through a wedge prism, pointed to the target, and received a view of his pointing hand only when it had contacted the curved surface on which the target was placed. In the second experiment, the subject received continuous exposure. That is, he saw the visual target and could see his hand continuously as he reached out to point to the target. In previous research (e.g., Cohen, 1967), significant adaptation of the exposed hand has been found for both terminal and continuous exposure conditions, while transfer of adaptation to the nonexposed hand has been found only in the terminal exposure condition.

Preceding the administration of the adaptation phase of each experiment, each subject performed a battery of eight pretests, which were identical for the two experiments. Since the hypothesis was that individual differences in adaptation performance would be in part attributable to differences in relevant abilities, the pretests were designed to provide various measures of eye abilities, hand abilities, and eye-hand coordination. Sets of pretest scores were used as predictors in multiple regression analyses with several measures of adaptation as dependent variables.

\section{GENERAL METHODS}

\section{Apparatus}

The apparatus was a modification of the localization equipment used in many previous studies of adaptation. The subject sat with his head in a chinrest and forehead restraint (the use of a bitebar interferes with EOG recording of eye movements). With his left eye occluded, the subject viewed with his right eye through either clear glass or a $10^{\circ}$ base right wedge prism, as the various conditions demanded. The subject made pointing responses under a shelf which contained a movable shield that could be arranged to prevent the subject's view of his hand altogether or to give continuous or terminal view of the pointing hand. A second shelf, parallel to the first, extended over the subject's head, and khaki cloth was stretched around the entire shelving in order to prevent the subject's view of the testing room. Visual targets were presented along the arc of a circle with radius of $50 \mathrm{~cm}$ and centered at the right eye.

Provision was made for recording both eye and hand movements in an ongoing nuanner. Eye movenents were recorded by means of a Beckman direct current electrooculography system. Previous evaluations of the accuracy of this system in our lab have shown that the $95 \%$ contidence interval around a given judgment is $\pm .75^{\circ}$. For each pointing response. the subject placed his foretinger on a trolley that ran out along a boom centered on an axle directly below the right eye. Five microswitches were spaced equally along the boom. The tirst switch was tripped as soon as the subject began to point, and the last was tripped as the subject's pointing finger contacted the arc on which the target was mounted. The axle of the boom extended to the floor. where it was connected to a potentiometer system. The output from the potentiometer appeared on one channel of the Beckman dynograph and thus provided a continuous record of the radial direction of the pointing hand with reference to the center of the apparatus. The microswitch output appeared on another dynograph channel. These two pointing channels thus provided a record of the radial direction of the pointing finger at each of five distances from the body.

\section{Pretests}

Each subject in both experiments performed an identical set of eight pretests. The pretests were presented in the order in which they are listed, and they were performed with the left eye occluded and the right eye viewing through clear glass.

Pretest 1: Fixating target. The subject was shown a point of light directly ahead of him in an otherwise dark environment and was directed to tixate the light for $30 \mathrm{sec}$. The score was the number of eyc movements with a lateral extent greater than $1^{\circ}$.

Pretest 2: Fixating an imaginary target in the dark. The subject was asked to maintain his tixation at straight ahead in a completely dark environment for $30 \mathrm{sec}$. Again, the score was the number of eye movements greater than $1^{\circ}$.

Pretest 3: Pointing to visual targets. A small light was placed at various azinuth target positions. $\pm 5^{\circ}$ and $\pm 10^{\circ}$ from the straight ahead. The subject was asked to fixate the target, then point to it with his unseen right hand. Each target position was used twice, for a total of eight trials. The series was then repeated with the subject using his left hand. The pointing data were used to assess the accuracy of pointing to visual targets with each hand: mean unsigned error and standard deviation were calculated for each hand. The eye-position data were used to generate the calibration functions needed for assessment of eye position in later tasks.

Pretest 4: Looking at felt hand position. The experimenter moved the subject's right forefinger to a target position and instructed the subject to look, in the dark, at where he felt his hand to be. The sane target positions were used as in Pretest 3 . After the right hand was used as a target, the series was repeated with the left foretinger as target. The mean unsigned error of aligning the eyes to the felt position of the hand and the standard deviation of the looking responses were calculated separately for each hand.

Pretest 5: Eye and hand tracking of a moving visual target. A small light was turned on $10^{\circ}$ to the subject's right of straight ahead. The subject looked at it and pointed at it with his right hand, then tracked it with both eye and hand as it was moved by the experimenter through straight ahead to $10^{\circ}$ to the subject's left, then back to $10^{\circ}$ to the right. The light was moved at a rate of approximately $5^{\circ}$ sec. The same procedure was then done with the left hand. with the target moving from $10^{\circ}$ left to $10^{\circ}$ right and back. Six measures were taken from this task, the number of times that each hand stopped in tracking the continuous movement of the target, the summed unsigned errors of pointing at the midpoint and the endpoint of the movement for each hand, and the number of eye discontinuities (the number of times the eye broke its smooth tracking of the target) separately for each hand's trial.

Pretest 6: Eye tracking of the unseen hand. The right, then the 
Table 1

Group Results on Pretest Measures $(\mathrm{N}=48)$

\begin{tabular}{|c|c|c|c|}
\hline Pretest & & Mean & SD \\
\hline \multicolumn{4}{|c|}{ A. Eye measures } \\
\hline 1 & Number of eye movements (target) & 7.1 & 5.5 \\
\hline 2 & Number of cye movements (no target) & 14.8 & 10.3 \\
\hline 5 & Number of eye movement episodes ( $R$ hand) & 5.4 & 3.2 \\
\hline 5 & Number of eye movement episodes ( $\mathrm{L}$ hand) & 6.4 & 3.9 \\
\hline 7 & Accuracy of reproduced eye position & $5.3 \mathrm{deg}$ & $3.4 \mathrm{deg}$ \\
\hline 7 & Variability of reproduced eye position & $6.1 \mathrm{deg}$ & $4.1 \mathrm{deg}$ \\
\hline \multicolumn{4}{|c|}{ B. Hand measures } \\
\hline 3 & Accuracy of pointing ( $R$ hand) & $3.5 \mathrm{deg}$ & $1.7 \mathrm{deg}$ \\
\hline 3 & Accuracy of pointing ( $L$ hand) & $3.4 \mathrm{deg}$ & $1.8 \mathrm{deg}$ \\
\hline 3 & Variability of pointing ( $R$ hand) & $2.9 \mathrm{deg}$ & $1.3 \mathrm{deg}$ \\
\hline 3 & Variability of pointing ( $L$ hand) & $3.0 \mathrm{deg}$ & $1.3 \mathrm{deg}$ \\
\hline 5 & Number of hand stops ( $R$ hand) & 9.9 & 3.0 \\
\hline 5 & Number of hand stops ( $L$ hand) & 8.9 & 2.9 \\
\hline 5 & Hand error (middle + end) $(\mathrm{R}$ hand) & $8.0 \mathrm{deg}$ & $4.5 \mathrm{deg}$ \\
\hline 5 & Hand error (middle + end) $(\mathrm{L}$ hand $)$ & $7.6 \mathrm{deg}$ & $5.0 \mathrm{deg}$ \\
\hline 8 & Accuracy of reproduced hand positions ( $R$ hand) & $3.4 \mathrm{deg}$ & $1.5 \mathrm{deg}$ \\
\hline 8 & Accuracy of reproduced hand positions ( $\mathrm{L}$ hand) & $4.0 \mathrm{deg}$ & $1.7 \mathrm{deg}$ \\
\hline 8 & Variability of reproduction ( $R$ hand) & $4.1 \mathrm{deg}$ & $2.3 \mathrm{deg}$ \\
\hline 8 & Variability of reproduction (L hand) & $4.3 \mathrm{deg}$ & $2.1 \mathrm{deg}$ \\
\hline \multicolumn{4}{|c|}{ C. Eye-hand measures } \\
\hline 4 & Accuracy of eye to hand ( $R$ hand) & $6.9 \mathrm{deg}$ & $3.7 \mathrm{deg}$ \\
\hline 4 & Accuracy of eye to hand (L hand) & $5.4 \mathrm{deg}$ & $3.6 \mathrm{deg}$ \\
\hline 4 & Variability of eye to hand ( $R$ hand) & $5.4 \mathrm{deg}$ & $3.4 \mathrm{deg}$ \\
\hline 4 & Variability of eye to hand ( $L$ hand) & $4.4 \mathrm{deg}$ & $2.2 \mathrm{deg}$ \\
\hline 6 & Number of eye discontinuities in tracking hand ( $R$ hand) & 15.1 & 5.8 \\
\hline 6 & Number of eye discontinuities in tracking hand ( $L$ hand) & 14.8 & 6.1 \\
\hline 6 & Eye-to-hand error (begin + middle + end) ( $R$ hand) & $30.8 \mathrm{deg}$ & $16.9 \mathrm{deg}$ \\
\hline 6 & Eye-to-hand error (begin + middle + end) (L hand) & $28.9 \mathrm{deg}$ & $13.7 \mathrm{deg}$ \\
\hline
\end{tabular}

lett hand was used as the target in a completely dark visual cmironment. The subject's hand was moved by the experimenter through the same path used in Pretest 5 . The subject's task was to cre-lrack the hand ats it moved. The measures taken from this pretest were the number of discontinuities in the eye-movement tracti, separately for each hand target, and the eye-to-hand errors summed wer the starting point, the haltway extreme point, and the end point of the movement. again separately for each hand.

Pretest 7: Replicating an eye position. The experimenter placed the target light at a target location, and the subject fixated it for a second. Then the light was turned off and the subject was instructed to look off to one side. then to look back at the place where the light had been. The same four target positions were used as in previous pretests. The mean unsigned error was tabulated, as was the standard deviation of localizations.

Pretest 8: Replicating a hand position. The experimenter moved the subject's right hand to a target position, held it there for a second, then moved it off to one extreme. The subject's task was to move his hand back to the target position. The visual environment was completely dark. Each of the four target positions was used once with each hand, and mean unsigned error and standard deviation were calculated for each hand.

In all, 26 submeasures were taken from the eight pretests. Complete pretest analysis was not possible for 2 of the 50 subjects. For the remaining 48 subjects, the means and standard deviations for each of the 26 submeasures appear in Table 1.

lle purpose in conductung the pretests wals to meatsure subject abilites that might be important in accounting for variance in measures of ataptation. The submeatsures were classified into cight abilits caltegories.

(1) Fae Acculacy. Mean unsigned error and standard deviation frum l'rescist 7.

(2) Hexe Smondhnes. Pretests 1 and 2; eye discontinuity meatsures
Imu Preks 5; eye dixcontinuity measures from Pretest 6.

(3) Right-ltand Pointing. Mean unsigned error and standard desiation of pointing by the right hand in Pretest 3; sum of unsigned crrors of right-hand pointing in Pretest 5.

(4) Leli-Hand Pointing. Paratel to Right-Hand Pointing.

(5) Right-Hand Proprioception. Mean unsigned error and sandatid deviation of directing the eyes to the right hand in Pretess 4: mean unsigned error and standard deviation of righthand pertormance in Pretest 8; summed unsigned errors of directing the eyes to the right hand in Pretest 6.

(6) Lefi-Hand Proprioception. Parallel to Right-Hand Proprioception.

(7) Right-Hand Smoothness. Number of right-hand discontinuities in tracking visual target in Pretest 5.

(x) Leli-Hand Smoothness. Paralled to Right-Hand Smoothness.

thus there were three types of categories, proprioception (representing afferent abilitics). response (representing efferent abilities). and smoothness. It should be noted that the Eye Snoothness and Hand Snoothness categories were parallel, but that the Eye Aceuracy category included both afferent and efferent componcints. while the afferent and efferent components were sepalrated into distinct categories for the hands. It should also be moted that several of the pretest submeasures could have been clissilied in other ways than they were. The most difticult decision was the classilication of Pretest 8 . replicating a hand position. which incolved both proprioception and pointing components. The Pretest 8 submeasures showed a stronger pattern of corretations with the other proprioceptive submeasures than with the other pointing subncasures. and so the pretest was included in the proprieceptive categeries. It may be noted from Table 2 that the correlations of the pointing with the proprioceptive categories were not signiticant. For other submeasures which involved two components and thus might have been classified in either of two 
Table 2

Correlation Matrix for Eight Ability Categories $(N=48)$

\begin{tabular}{lccccccc}
\hline & EyeAc & EyeSm & RHPt & LHPt & RHPro & LHPro & RHSm \\
\hline Eye accuracy & - & & & & & & \\
Eye smoothness & $.31^{*}$ & - & & & & & \\
R hand pointing & .01 & .15 & - & & & & \\
L hand pointing & -.01 & -.01 & $.54^{* *}$ & - & & & \\
R hand proprioception & $.37^{* *}$ & .04 & .11 & .11 & - & & \\
L hand proprioception & .17 & -.16 & .01 & .24 & $.40^{* *}$ & - & \\
R hand smoothness & -.15 & $.28^{*}$ & -.10 & .21 & $-.34^{*}$ & -.19 & $-79^{* *}$ \\
L hand smoothness & -.16 & .19 & -.02 & .05 & -.18 & -.05 & \\
\hline
\end{tabular}

ability categories, the decisions were typically not difficult because one component was clearly more contributory to performance than the other. For example, in Pretest 3, pointing to a visual target, an error could be due to misperceiving the target location because of inadequate eye movements, or to in accuracy of the hand in pointing to the target. The Pretest 3 submeasures were included in the hand-pointing categories on the grounds that the errors in directing the eyes to a small visual target are trivial when compared to the errors of pointing to a visual target.

Table 2 shows the pattern of correlations among the eight ability category scores. Several points are evident from the data in the table. First, the Eye Accuracy and Eye Smoothness categories were not independent of one another. Second, there were strong correlations between the right- and left-hand measures of smoothness, pointing, and proprioception. Third, the correlations between smoothness, pointing, and proprioception categories within the right-and the left-hand divisions were generally not signilicant, suggesting that these various categories might be expected to account for separate portions of variability in a dependent variable on which they are regressed.

Data from the 26 pretest submeasures were converted to z scores, and an ability index was formed for each subject for each category by summing his z scores for the submeasures in that category. thus. for each subject. there were eight indices, one representing his performance in each ability category. It was hypothesized that the ability indices. as assessed in the pretest phase, would contribute to variability in the dependent measures taken in the adaptation phase of the experiments.

\section{EXPERIMENT I: TERMINAL EXPOSURE CONDITION}

\section{Method}

In the terninal condition, a visual target subtending $.5^{\circ}$ was placed at the $100^{\circ}$ position, so that it appeared at straight ahead, or $90^{\circ}$. when viewed through the base-right prism. The subject was instructed to point to the target by placing his right forefinger on the trolley and pushing it out until his finger contacted the Plexiglas arc on which the target was mounted. The experimenter instructed the subject to take about 1.5 to 2 sec to make the pointing motion, then to retract the hand after 1 sec. The movable shield blocked the subject's view of his hand and the boom until his finger contacted the Plexiglas arc. The subject was not allowed to correct his response while the finger was in view. He pointed repeatedly at the target for a maximum of 20 trials. If the subject made five consecutive responses within $2^{\circ}$ of the visual target position, he was then given three additional trials and the adaptation phase was terminated. On each trial, the hand boom was started alternately $10^{\circ}$ to the subject's right or $10^{\circ}$ to his left of the previous response.

The adaptation task just described was the third in the series of six tasks needed to assess adaptation. Each of 25 subjects performed the six tasks in the same order. In Task 1, the subject peinted with his right hand at each of six visual targets. The shield was arranged to cover the hand completely, so that the subject received no information about the correctness of his response. Task 2 was the same as Task 1 , except that the subject used his left hand. Thus, Tasks 1 and 2 provided measures of the accuracy of pointing with right and left hands before adaptation. Task 3 was the adaptation task, already described. Task 4 consisted of four trials pointing with the left hand, with no visual feedback. Comparison of Task 4 with Task 2 thus provided a measure of the shift in pointing of the left (nonad apted) hand. Task 5 was included to regain any loss of adaptation of the right hand suffered during the delay involved in Task 4 . It consisted of a maximum of 12 right-hand adaptation trials, with terminal teedback as in Task 3. Task 6 consisted of four trials pointing with the right hand, with no feedback. Comparison of Task 6 with Task 1 thus provided a measure of right-hand adaptation. (Following these six tasks, additional tasks were administered to assess the course of adaptation back to conditions of zero visual displacement, but these data will not be discussed here.)

\section{Results}

Group results. A graphic representation of the group results appears in Figure 1. The ordinate is in degrees: $90^{\circ}$ is really straight ahead, but the subject had to point to his right, to $100^{\circ}$, to hit the target. The mean response shifted rapidly over the early trials. Criterion was defined for each subject as the first of any set of four trials on which the response was within $2^{\circ}$ of the target. The mean trials to criterion was 10.3 , standard deviation 6.6.

Another way to assess adaptation is to compare performance in no-feedback pre- and postadaptation conditions of pointing to visual targets. The horizontal arrows at the ordinate indicate the preadaptation performance for right and left hands (Tasks 1 and 2). The heavy vertical arrows represent the net change in pointing from pre- to postadaptation for right and left hands (i.e., Task 6 -Task 1, and Task 4-Task 2). The mean shift by the right hand was $7.8^{\circ}$, standard deviation $3.0^{\circ}$, while the mean shift by the left hand was $3.9^{\circ}$. standard deviation $3.8^{\circ}$. The adaptation as assessed in this way was significantly greater than zero for both hands, and the right-hand shift was significantly greater than the left-hand shift.

Multiple regression analyses. Stepwise multiple regression analyses were used to assess the relative contribution of each of the eight ability indices to the variance in each of several dependent measures of 
adaptation. Multiple regression as a data analytic technique can be used to assess the suitability of descriptive models. The stepwise procedure first selects the predictor that is most highly correlated with the dependent variable. In each subsequent step, that predictor is found which makes the next best prediction, that is, explains a new source of variance not overlapping those already determined. The importance of each predictor's contribution to the explanation of the dependent variable is best represented as the " $R^{2}$ added" $\left(\Delta R^{2}\right)$ by the predictor.

The dependent measures included the number of trials to criterion in the adaptation phase, the amount of right-hand adaptation, the amount of left-hand transfer, and the proportion of left-hand shift relative to right-hand adaptation. The measure of right-hand adaptation was the ratio of demonstrated adaptation to the total amount possible, given the individual subject's right-hand preexposure performance or constant error. That is, right-hand adaptation = $\left(\overline{\mathrm{X}}_{\mathrm{T} 6}-\overline{\mathrm{X}}_{\mathrm{T} 1}\right) /\left(100^{\circ}-\overline{\mathrm{X}}_{\mathrm{T} 1}\right)$. The measure of left-hand transfer was the ratio of demonstrated shift to the total anount possible, again taking the subject's preadaptation performance into account. That is, left-hand adaptation $=\left(\overline{\mathrm{X}}_{\mathrm{T} 4}-\overline{\mathrm{X}}_{\mathrm{T} 2}\right) /\left(100^{\circ}-\overline{\mathrm{X}}_{\mathrm{T} 2}\right)$. The following ratio was calculated to represent the proportion of left-hand shift relative to the right hand's adaptation: left-hand proportion = $\left(\overline{\mathrm{X}}_{\mathrm{T} 4}-\overline{\mathrm{X}}_{\mathrm{T} 2}\right) /\left(\overline{\mathrm{X}}_{\mathrm{T} 6}-\overline{\mathrm{X}}_{\mathrm{T} 1}\right)$.

The multiple correlation of the five pretest ability indices (excluding the left-hand indices) with the trials to criterion measure was .39. The indices accounted for only $15 \%$ of the variance in the measure. Clearly, the pretest categories did not bear much relevance to the speed of adaptation.

For the right-hand adaptation measure, the ability categories accounted for about $29 \%$ of the variance in the dependent measure $(\mathrm{R}=.54)$. The most important category was Right-Hand Smoothness $\left(\Delta \mathrm{R}^{2}\right.$ $=.15)$, followed by Eye Smoothness $\left(\Delta \mathrm{R}^{2}=.07\right)$ and Eye Accuracy $\left(\Delta \mathrm{R}^{2}=.04\right)$. The pattern of correlations between the pretest submeasures and the dependent variable showed that the better the hand abilities were, the less adaptation occurred, while the better the eye abilities were, the greater the adaptation. The results make good sense if we consider that the subject is confronted with a problem to solve (missing the visual target) in the early adaptation trials, and that he has two sources of information available (visual and proprioceptive) to help him solve the problem. The better his eye abilities are and the worse his hand abilities are, the greater the subject's tendency is to solve the problem by adjusting his hand behavior to fit the visual information, and the more adaptation he shows. Conversely, the better his hand abilities are, the less tendency he has to solve the problem by adjusting his

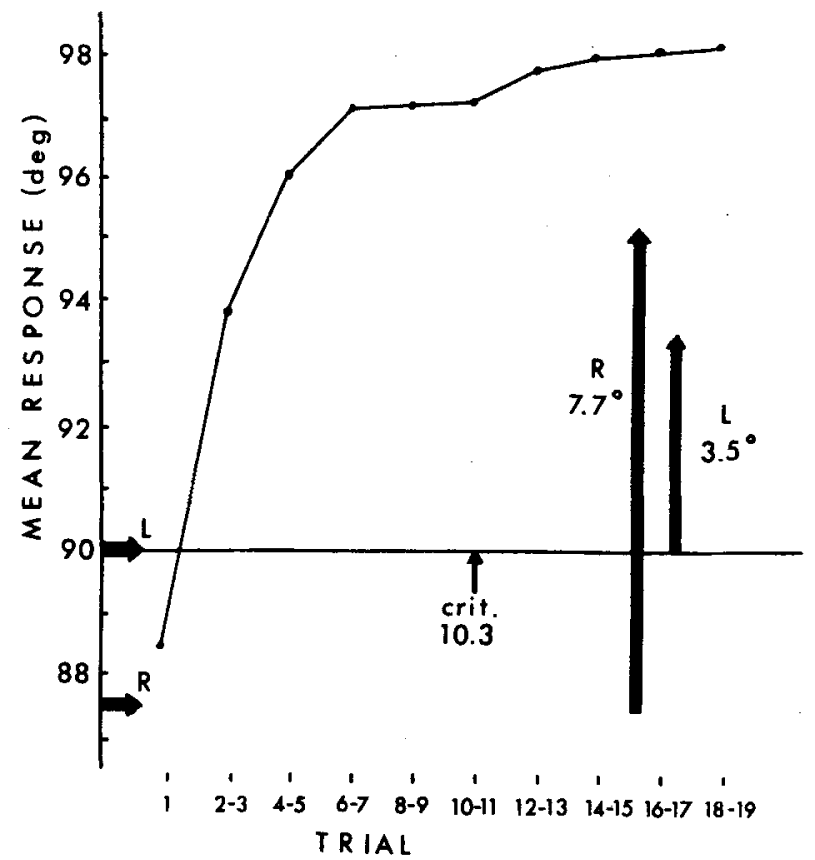

Figure 1. Growth of adaptation, terminal condition.

hand behavior to suit the visual information, and the less adaptation he shows.

For the left-hand dependent measures, all eight ability indices were included as predictors, since it seemed likely that some right-hand abilities might influence the amount of left-hand shift. For the left-hand adaptation measure, the ability indices accounted for $33 \%$ of the variance $(R=.58)$. The important indices were Eye Accuracy $\left(\Delta R^{2}=.10\right)$, Left-Hand Proprioception ( $\left.\Delta \mathrm{R}^{2}=.06\right)$, Left-Hand Pointing $\left(\Delta R^{2}=.05\right)$, Left-Hand Smoothness $\left(\Delta R^{2}=\right.$ $.03)$, and Right-Hand Pointing $\left(\Delta R^{2}=.03\right)$. The directions of these relationships were the same as in the analysis of left-hand proportion, discussed below.

The left-hand adaptation measure disregards what may be an important determinant of left-hand adaptation, namely the amount of adaptation shown by the right hand. It may be unreasonable to equate the absolute magnitudes of left-hand adaptation for two subjects, one of whom showed a lot of right-hand adaptation and the other of whom showed very little. A more appropriate dependent measure for the left-hand effect is one that takes into account the magnitude of the right hand's adaptation. The left-hand proportion measure served this purpose by representing how completely the right hand's effect (however large or small it was) transferred to the left hand. The ability indices accounted for $60 \%$ of the variance in this dependent measure $(R=.78)$. Eye Accuracy $\left(\Delta R^{2}=.40\right)$ was the greatest contributor to the measure, followed by Left-Hand Proprioception 
$\left(\Delta \mathrm{R}^{2}=.08\right)$ and Left-Hand Smoothness $\left(\Delta \mathrm{R}^{2}=.07\right)$. The pattern of correlations between the pretest submeasures and the dependent variable showed that the better the eye accuracy was. the less transfer occurred. and that the smoother and more accurate the left hand wals. the more transfer occurred.

The results from the analyses of transfer apparently create a puzzle. The hand and eye abilities were related to the dependent measures in an inverse manner, as was the case for right-hand adaptation. However. the directions of the relationships were reversed. Good eye abilities were related to a large right-hand adaptation but to a small left-hand transfer. Good hand abilities were related to small right-hand adaptation but to a large left-hand transfer.

There are two distinct points to be considered in explaining left-hand transfer. One is the amount of adaptation shown by the right hand. Then, if this amount of adaptation is considered to be available for transfer, the second question is how to account for the amount of adaptation that actually does transfer to the left hand. A study by Cohen (1967) bears on the tirst question. Cohen found a significant intermanual transfer of adaptation under conditions of terminal exposure, although the magnitude of the transfer was significantly less than the magnitude of the adaptation shown by the exposed hand. Cohen suggested that the adaptation shown by the exposed hand consists of two components, one a shift in the proprioceptive position of the exposed arm, and the other a shift in visual direction of gaze. The proprioceptive arm shift component of the adaptation is local to the adapted arm and therefore is not available for transfer, but the visual shift component is general and may exert an effect on the behavior of the contralateral hand. In the present study, as in Cohen's, it was unfortunately not possible by direct means to separate the right hand's adaptation into proprioceptive arm shift and visual shift components. Less directly, however, it may be argued, on the basis of Cohen's formulation, that if it is only the visual shift component that is available for transfer, then those subjects who showed more left-hand transfer must have acquired more of the visual shift component than the subjects who showed very little left-hand transfer. The results do provide some support for this formulation. The correlation between the Eye Accuracy index (on which a high score means poor accuracy) and left-hand transfer was .63, indicating that those subjects with better eye accuracy showed less left-hand shift.

With respect to the second question, it should be noted that there are other factors besides the amount of visual shift adaptation that may affect the magnitude of shift shown by the contralateral hand. Specitically, the abilities of the left hand should be involved. In fact, both Left-Hand Proprioception $\left(\Delta \mathrm{R}^{2}=.08\right)$ and Left-Hand Smoothness $\left(\Delta \mathrm{R}^{2}=.07\right)$ contributed substantially to variability of left-hand transfer. As already noted, both relations showed that the better the left-hand abilities were. the more transfer occurred. How may these results be explained? Consider the implications of the visual shift that is hypothesized to develop during right-hand adaptation. The effect of the visual shift is to produce a new frame of reference, one that is shifted some number of degrees laterally (to the subject's right, in the present situation). Thus, in Task 4 , where the subject saw a visual target and had to point to it with his left hand. the apparent position of the target was shitted laterally from its apparent position before adaptation. The better the subject's left-hand abilities are, the better he should be able to point where he wants to, namely to the position of the visual target within the new, shifted frame of reference. The fact that those subjects with better left-hand abilities showed more left-hand transfer thus makes sense within this formulation.

It should be noted that these explanations are based on post hoc reasoning. Logically, the weakest part of the argument is that there was no basis, independently of the left-hand transfer results, to assess the magnitude of the visual shift component of adaptation acquired during adaptation of the right hand. Further experimentation, in which the visual shift component is independently assessed, is necessary.

\section{EXPERIMENT II: CONTINUOUS EXPOSURE CONDITION}

\section{Method}

In the continuous adaptation condition, the experimental situation was identical to that described for the terminal condition except in Taisks 3 and 5. In Task 3, the adaptation exposure task. the shicld wats removed so that the subject could see his hand during the entire pointing movement. with the exception of the initial 3-t in., where the subject s hand was hidden by the prism mount. Sis blocks of trial, were given in lask 3. A block consisted of four continuous exposure trials. followed by two test trials where the shichl was arranged to prevent visual feedback completely. The subject was instructed to take 1.5 to 2 sec to point, then pull his hand hack completely, then point again with a delay of only a second or two. The no-feedback trials in each block followed in the same timing sequence, since the experinenter could slide the shield into place during the short delay. In Task 5, two blocks of trials were given. 'lasks 1. 2. 4, and 6 were the same as in the terminal condition.

Twenty-lise native college sludents served as subjects. None had patricipated in the previous experinent.

\section{Results}

Group results. A graphic representation of the group results appears in Figure 2. Again, the ordinate is in degrees, with $90^{\circ}$ straight ahead. The subject had 
to point to his right, to $100^{\circ}$, to hit the target. Each datta point represents the mean of the two no-feedback trials in a block in Task 3 . The position of the first dat a point show's that considerable shift had occurred as a result of the first four adaptation trials. Criterion was detined for each subject as the first block on which the two no-feedback trials averaged within $2^{\circ}$ of the target position, if the corresponding trials on the succeeding block were also within $2^{\circ}$. Mean trials to criterion was 5.3, standard deviation 4.2.

Magnitude of adaptation was also assessed by comparison of Tasks 6 and 1 , as in the terminal condition, and left-hand transfer was assessed by comparison of Tasks 4 and 2. The horizontal arrows at the ordinate represent the preadaptation performance for right and left hands (Tasks 1 and 2), and the heavy vertical arrows represent the net change from pre- to postadaptation for right and left hands. The mean shift by the right hand was $6.8^{\circ}$, standard deviation $3.0^{\circ}$, while the mean shift by the left hand was $0.7^{\circ}$, standard deviation $1.8^{\circ}$. The mean left-hand shift was not significantly different from zero. The failure to find a significant left-hand effect provides corroboration of the results of Cohen (1967), who reported no transfer of adaptation to the contralateral hantd under conditions of continuous exposure.

Multiple regression analyses. The same ability indices were calculated on the basis of pretest performance as for the terminal condition. The ability indices were again used as predictors in several stepuise multiple regression analyses, one for each of the same dependent measures used in the terminal condition analyses.

The multiple correlation of five ability indices (excluding the left-hand indices) with the right-hand adaptation measure was .54; thus the indices accounted for $29 \%$ of the variance in the measure. Right-Hand Pointing contrihuted the major part of the variance $\left(\Delta R^{2}=.17\right)$, followed by Right-Hand Proprioception $\left(\Delta \mathrm{R}^{2}=.05\right)$, Eye Smoothness $\left(\Delta R^{2}=.03\right)$, and Right-Hand Smoothness $\left(\Delta R^{2}=\right.$ $.03)$. The direction of the correlations indicated that subjects with better right-hand abilities showed less adaptation, and that subjects with better Eye Smoothness showed more adaptation. These relationships were parallel to those found in the terminal condition, in that better eye abilities were associated with more adaptation, while better hand abilities were related to less adaptation.

Similar results were found for the trials to criterion measure. The five ability indices accounted for $58 \%$ of the variance in this measure $(R=.76)$. The most important indices were Right-Hand Smoothness $\left(\Delta \mathrm{R}^{2}=.30\right)$ and Right-Hand Pointing $\left(\Delta \mathrm{R}^{2}=.26\right)$. The direction of the relationships indicated that subjects with better hand abilities took more trials to reach criterion.

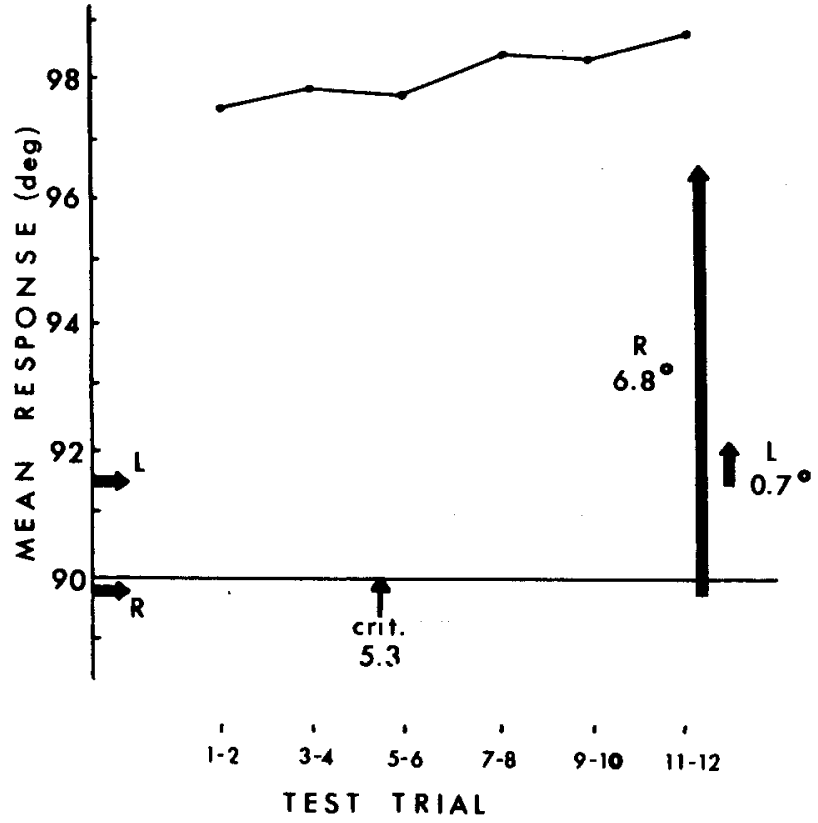

Figure 2. Growth of adaptation, continuous condition.

Multiple regression analyses were conducted using both left-hand dependent measures. The ability indices accounted for only $14 \%$ of the variance in the left-hand adaptation measure, and for only $17 \%$ of the variance in the left-hand proportion measure. The failure of the categories to account for substantial variance was not surprising in view of the lack of a significant shift by the left hand.

Despite the fact that the left-hand transfer measures did not show effects significantly greater than zero for the continuous exposure group, there were some subjects who did show evidence of transfer. In lact. left-hand transfer, expressed in degrees of shift, ranged from $-1.7^{\circ}$ to $+4.5^{\circ}(+$ indicates a shift in the direction of adaptation). In an earlier paper (Warren \& Platt, Note 1), we suggested that some subjects in a continuous exposure condition might effectively be in a terminal condition. That is, despite the fact that the hand is visible during its pointing movement, the subject might retain fixation on the visual target and not really gain the advantage of the continuous exposure. Such a subject might be expected to show intermanual transfer. To test this formulation, we measured the frequency of lateral eye movements during the pointing movements in the first block of four adaptation trials in Task 3, the ataptation phase. The correlation of this eyemovement measure with the amount of left-hand shift (in degrees) was $-.49(\mathrm{p}<.02$, df $=20)$. That is, those subjects who made fewer movements during the early adaptation trials showed more transfer of adaptation than those whose eyes were more active 
during adaptation. The question also occurs whether the relationship between left-hand transfer and eye movements is one that is specific to the adaptation situation itself or is more general. Would the amount of left-hand transfer be correlated with an eye-activity measure taken independently of the adaptation situation? A frequency-of-eye-movement measure was taken from Task 1 of the adaptation phase, where the subject pointed with his right hand to a visual target without seeing his hand. The correlation of this measure with the magnitude of left-hand shift was $-.54(\mathrm{p}<.01, \mathrm{df}=22)$ : left-hand shift was greater for subjects who showed relatively few eye movements in a situation independent of the adaptation phase.

It is unwise to draw causal conclusions from these results, since it is possible that some third factor influenced both amount of left-hand shift and frequency of eye movements. However, the data do not contradict the notion that subjects who show less eye activity during adaptation are effectively in an adaptation condition that involves terminal rather than continuous exposure. Further research, in which eye activity is influenced by instruction or some other experimental manipulation, might provide a definitive test of this notion.

\section{DISCUSSION}

The group results from the two prism-adaptation conditions reported here did not differ markedly from those of other prism work. In the terminal condition, the adaptation by the exposed hand averaged about $60 \%$ of the amount possible, while the unexposed hand showed an average adaptation of about $40 \%$. In the continuous condition, the exposed hand showed an average adaptation of about $65 \%$, and that of the unexposed hand averaged less than $10 \%$ and was not significantly greater than zero.

In recent years, several investigators have argued that it is oversimplified to consider adaptation to prisms simply as a shift in felt hand position or a shift in some aspect of visual direction. Rather, adaptation consists of at least two separate components which depend on different sources of information and which may develop at different rates. The present experiments corroborate the complexity of the adaptation process. In fact, the results demonstrate that the strength of the various components of adaptation depends not only on different sources of information, but on different abilities of subjects to make use of the available information. In the terminal exposure condition, more adaptation was found for subjects who had good eye abilities and less adaptation was found for subjects who had good hand abilities. Apparently, subjects with better eye abilities tended to solve the problem of missing the visual target by depending more on the visual information. and they therefore showed more adaptation. The subjects with better hand abilities did not have such a strong tendency to rely on the visual information and therefore did not adapt as much. The phenomenon of intermanual transfer of adaptation is also claritied by the present results. The subjects with better eye abilities apparently acquired less of the visual shift component of adaptation, the component that is thought to be available for intermanual transfer (Cohen, 1967). Furthermore, the amount of the available adaptation that actually did transfer depended partly on the abilities of the left hand. Those subjects with better left-hand abilities showed more transfer.

The results of the continuous exposure condition are also useful. The magnitude of initial adaptation by the right hand showed a pattern of dependence on eye and hand abilities that was similar to the pattern for the terminal condition, although the indices of eye abilities were less important in accounting for variance than they were in the terminal condition. Detailed analysis of the eye-activity characteristics of individual subjects supported the hypothesis that the occurrence of intermanual transfer in the continuous condition depends on the extent to which the subject actually makes use of the continuous view of his hand in the initial adaptation. Intermanual transfer of adaptation did occur for some subjects in the continuous condition, but it occurred for those subjects whose eye-behavior characteristics made the continuous condition effectively a terminal condition.

Most of the work on adaptation to prisms has implicitly taken the approach that a single explanation may be found that applies equally well to all subjects. This approach has been characteristic even of theories that consider adaptation to be a multicomponent process. We suggest not only that adaptation is a multicomponent process, but also that the relative importance of the components varies from subject to subject, not in a haphazard way but in a way that may in part be explained by reference to various perceptual abilities of the subjects. Adaptation to prisms may indeed be primarily a result of shift in felt hand position for some subjects, but primarily a result of shift in visual direction for other subjects. Adequate experimental treatment of this complexity requires consideration of not just the characteristics of the experimental situation, but also of individual differences in ability characteristics that exist independently of the experimental situation. 


\section{REFERENCE NOTE}

1. Warren, D. H., \& Platt, B. B. The subject: A neglected factor in recombination research. Paper delivered at the Conference on the Recombination Procedure as a Tool for the Study of Visual Perception, November 1973, University of Kansas.

\section{REFERENCES}

Confe, M. M. Continuous versus terminal feedback in prism aftereffects. Perceptual and Motor Skills, 1967, 24, 1295-1302. Harris, C. S. Perceptual adaptation to inverted, reversed. and displiced rision. Psychological Review, 1965, 72, 419.444.

HAY, J. C.. \& PiCk. H. L., JR. Visual and proprioceptive adaptation to ptical displacement of the visual stimulus.
Journal of Experimental Psychology, 1966, 71, 150-158.

Mclaughlin, S. C., \& Bower, J. L. Selective intermanual transfer of adaptive effects during adaptation to prism. Psychomomic Science, 1965, 3, 69-70.

Mclaughle, S. C., Rifkin, K. I., \& Webster, R. G. Oculomotor adaptation to wedge prisms with no part of the body seen. Perception \& Psychophysics, 1966, 1, 452.458.

Welch, R. B., \& RHOADEs, R. W. The manipulation of informational feedback and its effects upon prism adaptation. Canadian Joumal of Psychology, 1969, 23, 415-428.

(Received for publication June 20, 1974; revision received November 22, 1974.) 